\title{
A symmetric and an asymmetric model for the equal-sensation function in olfaction
}

\author{
LEIF T. SVENSSON \\ University of Umed, Umed, Sweden
}

\begin{abstract}
Data from various intramodel matching experiments in olfaction were analyzed with regard to a symmetric and an asymmetric model for the equal-sensation function. The asymmetric model was discussed in relation to the symmetric model. In all, 11 equal-sensation functions were investigated, and of these 9 were with different pairs of odorants. The following odorants were investigated: hydrogen sulfide, pyridine, dimethyl disulfide, and five odorants obtained by different combustion procedures of animal manure. It was found that the equal-sensation function can be written in the following asymmetric form:
\end{abstract}

$$
\phi_{\mathrm{i}}=\mathrm{b}_{\mathbf{i k}}^{\lambda} \cdot \phi_{\mathbf{k}}^{b_{i k}}, \psi_{\mathrm{i}}=\psi_{\mathbf{k}}
$$

or symmetric form:

$$
\phi_{\mathbf{i}}=\mathrm{C}^{1-\mathrm{b}_{\mathbf{i k}}} \cdot \phi_{\mathbf{k}}{ }^{\mathrm{b}_{\mathbf{i k}}}, \psi_{\mathbf{i}}=\psi_{\mathbf{k}},
$$

where $\phi_{\mathbf{i}}$ and $\phi_{\mathbf{k}}$ are stimuli expressed in multiples of respective absolute thresholds, $\lambda$ and $\mathrm{C}$ are general constants invariant of experimental matching method and matched attribute (perceived unpleasantness or intensity). The constants $\lambda$ and $\mathrm{C}$ were calculated both for group data and individual data. The asymmetric form of the equal-sensation function was interpreted in terms of relativity and the symmetric form in terms of measurement.

In psychological research, several matching experiments have been carried out. These experiments have concerned (1) matching between sensory modalities, i.e., cross-modality matching, and (2) matching within sensory modalities, i.e., intramodality matching. The experiments have been performed with mainly two types of attributes involved, (a) matching of perceived intensity and (b) matching of perceived pleasantness or unpleasantness. Examples of Class 1a matchings can be seen in a paper by S. S. Stevens (1960), for several pairs of modalities. Investigations of Class $2 \mathrm{a}$ are, for example, experiments in audition (e.g., Stevens, 1966), heterochromatic matching of saturation to saturation (Indow \& Stevens, 1966), for vibrotactile stimulation (Stevens, 1968), and for matching of linear aliphatic alcohols (Cain, 1969). Examples of Class 2b experiments can be found in audition for perceived noisiness (Kryter

This report is based on part of a doctoral dissertation submitted to the Psychological Laboratories, University of Stockholm. The investigation was supported by a grant from the University of Stockholm and by a grant to Dr. B. Berglund from the Swedish Council for Social Science Research. Thanks are extended to Mr. P. O. Olsson, Mr. S. Pettersson, and Mr. G. Rosén for assistance with the experiments. The author is indebted to Dr. Thomas Lindvall for lending him the facilities of the olfactory laboratory at the Karolinska Institute. The author's address: Department of Psychology, University of Umeá, S-901 87 Umeå, Sweden.
\& Pearsons, 1963; Reese, Kryter, \& Stevens, Note 1) and in olfaction for unpleasantness (Lindvall \& Svensson, 1974). In all these experiments, it was shown that a simple power function adequately describes the relation between a matching continuum and a reference continuum except for the experiments on noisiness in audition. For that case, the obtained equal-noisiness contours are more complicated (Stevens, 1970).

The equal-sensation function is of the basic form,

$$
\phi_{i}=a_{i k} \phi_{k}^{b_{i k}}, \psi_{i}=\psi_{k}, i, k=1, \cdots, n,
$$

where $\phi$ and $\psi$ represent physical and perceptual continua, respectively. The first index (i) represents the matching continuum, and the second (k), the reference continuum.

The matching experiments thus far performed have been concerned with the size of the exponent and continua matched. The exponent in the equalsensation function has also been predicted from exponents from magnitude estimation experiments (e.g., Stevens, 1966). However, a systematic study of the multiplicative constant in the equal-sensation function has been neglected in theorizing.

The present report is concerned with a synthesis of the findings in three experiments regarding matching of odorous substances. The synthesis takes into account the whole equal-sensation function. Data 
from two experiments dealing with applied air pollution research that have been published in other contexts will be used (Lindvall \& Svensson, 1974; Svensson \& Lindvall, 1974). In addition, a third experiment has been performed that will be reported here. From the two former experiments, only data relevant in the present context will be presented.

\section{PROBLEM}

The primary aim of this investigation is to find out if any simple function describes the relation between the multiplicative constant $\left(a_{i k}\right)$ and the exponent $\left(b_{i k}\right)$ in the equal-sensation function (see Equation 1). This relationship should be independent of odors matched in order to satisfy the criterion of parsimony and give interpretative meaning to the equalsensation function.

A scale whose permissible transformations are power transformations is called a log-interval scale because a logarithmic transformation of such a scale results in an interval scale (Krantz, Luce, Suppes, \& Tversky, 1971). In the equal-sensation function (Equation 1), the matching continuum is related to the reference continuum by a power transformation. The scale of the matched continuum is therefore a log-interval scale. If a general constant can be found, invariant of matched odors, attribute, and experimental matching method, describing the relationship between the multiplicative constant $\mathrm{a}_{\mathrm{ik}}$ and the exponent $b_{i k}$ in Equation 1, the scale values of the matched odorant ought to be on a higher level of measurement than log interval. This particular case seems not to have been considered by Krantz et al. (1971). One of the aims of the present study is to approach the problem according to some of the measurement properties of a relationship. Specifically, let $\mathrm{i}, \mathrm{j}$, and $\mathrm{k}$ be three continua and $\phi_{\mathrm{i}}, \phi_{\mathrm{j}}$, and $\phi_{\mathrm{k}}$ be physical intensities on these continua. Let $M$ be the matching relation. The matching relation, $\mathbf{M}$, is said to be transitive, symmetric, and reflexive according to the criteria:

Transitivity: If $\phi_{\mathrm{i}} \mathbf{M} \phi_{\mathrm{j}}$ and $\phi_{\mathrm{j}} \mathrm{M} \phi_{\mathrm{k}}$, then $\phi_{\mathrm{i}} \mathrm{M} \phi_{\mathrm{k}}$.

Symmetry: If $\phi_{i} M \phi_{j}$, then $\phi_{j} M \phi_{i}$.

Reflexivity: $\quad \phi_{\mathrm{i}} \mathbf{M} \phi_{\mathrm{i}}$.

If all three criteria are fulfilled, $M$ is said to be an equivalence relation. For such a relation, certain rules for the combination of exponents in the equalsensation function for different continua are given.

Another aim is to give a possible nonstatistical interpretation of the so-called regression effect described by Stevens and Greenbaum (1966). That is, when one continuum (i) is matched to another con- tinuum (k), the exponent, $b_{i k}$, obtained will not be equal to $1 / b_{k i}$, where $b_{k i}$ is the exponent that will be obtained when continuum $(k)$ is matched to continuum (i).

\section{Is the Equal-Sensation Function Symmetric or Asymmetric?}

Consider the equal-sensation function shown in Equation 1 and let stimuli be expressed in multiples of absolute thresholds. Two possible functions relating the exponent to the multiplicative constant are suggested. Both functions can be regarded as postulates (see, e.g., Hempel, 1966, pp. 7-11). It may be shown that one of the postulated functions is a good postulate, even if not true, by an empirical test and its theoretical advantageous consequences. In addition, both the postulated functions are of real simple form, lending them support according to the parsimony criterion considered in empirical science (Hempel, 1966).

Let

$$
a_{i k}=C^{1-b_{i k}}
$$

and

$$
a_{i k}=b_{i k}^{\lambda}
$$

be an exponential and a power function, respectively, describing the relationship between the multiplicative constant $\left(a_{i k}\right)$ and the exponent $\left(b_{i k}\right) . C$ and $\lambda$ are constants.

In Section A below, it will be shown that the equal-sensation function is transitive and symmetric under conditions according to Equation 2, and in Section B that it is neither transitive nor symmetric under conditions according to Equation 3.

Let us call the equal-sensation function according to Equation 2 the symmetric model. The asymmetric model will then, in analogy, be the equal-sensation function according to Equation 3 that may explain regression phenomena (see under Section $C$ below). For this reason, the asymmetric model has higher explanatory value than the symmetric model.

In order to show the characteristics of the two models, the following four functions have to be formulated. Let $\mathrm{i}, \mathrm{j}$, and $\mathrm{k}$ be three continua and

$$
\begin{aligned}
\phi_{i}=a_{i k} \phi_{k} b_{i k} & \left(\phi_{i} M \phi_{k}\right) \\
\phi_{i}=a_{i j} \phi_{j} b_{i j} & \left(\phi_{i} M \phi_{j}\right) \\
\phi_{j}=a_{j k} \phi_{k} b_{j k} & \left(\phi_{j} M \phi_{k}\right) \\
\phi_{k}=a_{k j} \phi_{j} b_{k j} & \left(\phi_{k} M \phi_{j}\right)
\end{aligned}
$$

be the four equal-sensation functions relating these continua to each other. 
(A) The symmetric model. It will be proven in this section that the equal-sensation function is transitive and symmetric under conditions according to Equation 2. From Equations 2, 5, and 6, we have

$$
\phi_{i}=C^{\left[1-b_{i j}+\left(1-b_{j k}\right) b_{i j}\right]_{\phi_{k}}\left[b_{j k} b_{k j}\right] .}
$$

Equation 8 can be reduced to

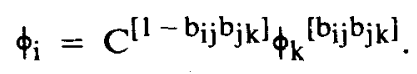

That is, from Equations 2, 4, and 9, we obtain by identification of exponents

$$
b_{i j} b_{j k}=b_{i j} \text {, (if } \phi_{i} M \phi_{j} \text { and } \phi_{j} M \phi_{k} \text { then } \phi_{i} M \phi_{k} \text { ). }
$$

Thus we have shown that the equal-sensation function is transitive under conditions according to Equation 2.

In order to show that the symmetry criterion is fulfilled, we proceed in an analogous way. From Equations 2, 6, and 7, we have

$$
\phi_{j}=C^{\left[1-b_{j k}+\left(1-b_{k j}\right) b_{j k}\right]_{\phi_{j}}\left[b_{k j} b_{j k}\right]} .
$$

Equation 11 can be reduced to

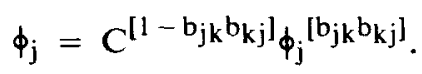

That is, under the assumption of reflexivity $\left(b_{j}=1\right.$, for $\phi_{\mathrm{j}} \mathrm{M} \phi_{\mathrm{j}}$ ), we obtain from Equation 12

$$
b_{j k} \cdot b_{k j}=1,\left(\text { if } \phi_{j} M \phi_{k} \text { then } \phi_{k} M \phi_{j}\right) \text {. }
$$

Thus the equal-sensation function is also symmetric under conditions according to Equation 2.

(B). The asymmetric model. It will be proven in this section that the equal-sensation function is neither transitive nor symmetric under conditions according to Equation 3. From Equations 3, 5, and 6 , we have

$$
\phi_{i}=\left[b_{i j} b_{j k} b_{i j}\right]_{\phi_{k}} b_{i j} b_{j k} .
$$

That is, from Equations 3, 4, and 14, we obtain by identification of exponents

$$
\left.\begin{array}{l}
b_{i j}=b_{i j} b_{j k} b_{i j} \\
b_{i j}=b_{i j} b_{j k}
\end{array}\right\}
$$

It should be noticed that both these functions must be valid at the same time, i.e., only when $b_{i j}=1$ and $/$ or $b_{j k}=1$. It follows that the equal-sensation function is intransitive under conditions according to Equation 3. In the same way, we find from Equations 3,6 , and 7 that,

$$
\phi_{j}=\left[b_{j k} b_{k j} b_{j k}\right]^{\lambda} \phi_{j} b_{j k} \cdot b_{k j} .
$$

That is, under the assumption of reflexivity $\left(b_{j j}=1\right.$, $\phi_{\mathrm{j}} \mathbf{M} \phi_{\mathrm{j}}$ ), we obtain from Equation 16

$$
\left.\begin{array}{l}
1=b_{j k} b_{k j} b_{j k} \\
1=b_{j k} b_{k j}
\end{array}\right\}
$$

Again, both functions must be valid at the same time, i.e., only when $b_{j k}=1$ and/or $b_{k j}=1$. Thus, the equal-sensation function is also asymmetric under conditions according to Equation 3 .

(C) A comparison between the symmetric and asymmetric model. In this section, the so-called regression effect in psychophysical judgment, given a statistical explanation by Stevens and Greenbaum (1966), will be given a nonstatistical interpretation by a comparison of the symmetric and asymmetric model. Taking the natural logarithm of Equation 3, we obtain

$$
1 n a_{i j}=\lambda 1 n b_{i k} .
$$

And regarding the following limit

$$
\lim _{b_{i k} \rightarrow 1} \frac{\ln b_{i k}}{b_{i k}-1}=1 \text {, }
$$

we can write approximately, for $b_{i k}$ close to 1 ,

$$
a_{i k}=e^{\lambda\left(b_{i k}-1\right)},
$$

but Equation 20 is equivalent with Equation 2 and a comparison gives

$$
C=e^{-\lambda}
$$

In other words, the asymmetric equal-sensation function will approach the symmetric equal sensation function for $b_{i k}$ close to 1 and at the limit they are connected according to Equation 21.

The expression $1-b_{i k}$ is associated with the symmetric model, while $1 \mathrm{n}\left(1 / \mathrm{b}_{\mathrm{ik}}\right)$ is associated with the asymmetric model. It was also shown earlier that the symmetric model is symmetric and transitive and that the asymmetric model is asymmetric and intransitive. The concrete meaning is obvious from Table 1 , as $1-b_{i k}$ is an underestimate of $\ln \left(1 / b_{i k}\right)$. The so-called regression effect is assumed to be associated with the ratio $1-b_{i k} / 1 n\left(1 / b_{i k}\right)$. From the table it can be seen that the effect is enhanced as $b_{i k}$ decreases from 1 .

In order to show some properties of the asymmetric and symmetric models, let us rewrite them in the following way: 
Table 1

The Regression Effect: A Comparison Between the Symmetric and Asymmetric Model

\begin{tabular}{cccc}
\hline$b_{\mathbf{i k}}$ & $1-\mathrm{b}_{\mathbf{i k}}$ & $1 \mathrm{n}\left(1 / \mathbf{b}_{\mathbf{i k}}\right)$ & $\frac{1-\mathbf{b}_{\mathbf{i k}}}{1 \mathrm{n}\left(1 / \mathrm{b}_{\mathbf{i k}}\right)}$ \\
\hline .90 & .10 & .10 & 1.00 \\
.80 & .20 & .22 & .91 \\
.70 & .30 & .36 & .84 \\
.60 & .40 & .51 & .79 \\
.50 & .50 & .69 & .72 \\
\hline
\end{tabular}

and

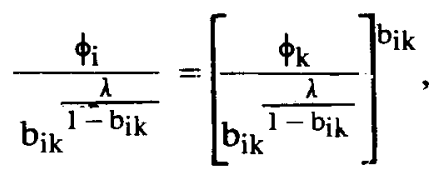

$$
\frac{\phi_{\mathrm{i}}}{\mathrm{C}}=\left[\frac{\phi_{\mathrm{k}}}{\mathrm{C}}\right]^{\mathrm{b}_{\mathrm{ik}}},
$$

where stimulus intensities $\phi$ are expressed relative to absolute threshold, i.e., where $S$ is stimulus concentration in any unit and $S_{o}$ absolute threshold in the same unit.

\section{Experimental Threshold}

Let us now introduce the construct experimental threshold or experimental adaptation level, $\mathrm{S}_{\mathrm{e}}$. It is assumed that the experimental threshold for the two models is defined by

$$
\mathrm{S}_{\mathrm{eas}}=\mathrm{S}_{\mathrm{o}} \cdot \mathrm{b}_{\mathrm{ik}} \frac{\lambda}{1-\mathrm{b}_{\mathrm{ik}}}
$$

and

$$
\mathrm{S}_{\mathrm{es}}=\mathrm{S}_{\mathrm{o}} \cdot \mathrm{C} \text {, }
$$

respectively. The experimental threshold is suggested to be the pooled effect introduced in the experimental situation in analogy to the interpretation of the steepening of slope found for the psychophysical function near threshold (cf. Berglund, 1971).

The asymmetric model and its general implications. In Section B above, it was shown that, given the exponent of the equal-sensation function for two odors, $\mathrm{A}$ and $\mathrm{B}$, and the exponent for odor $\mathrm{B}$ and $\mathrm{a}$ third odor $C$, the exponent for odors $A$ and $C$ cannot be predicted. This property of intransitivity for the asymmetric model can be interpreted in terms of relativity, because percepts cannot be predicted and they will in this way be relative in the pure sense of the word. Every exponent relating two odors in an equal-sensation function is therefore unique and relative. However, a general constant, $\lambda$, was found that is invariant of experimental matching method, substances matched, and whether the matching concerned perceived unpleasantness or intensity.
From Equation 22, it follows that it may be meaningful to talk about a relative origin in the equalsensation plane. The coordinates for the relative origin $\left(\mathrm{O}_{\mathrm{r}}\right)$ are:

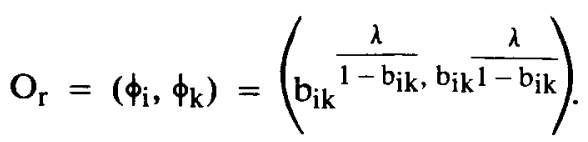

The relative origin is here expressed in the absolute constant, $\lambda$, and the relative exponent $b_{i k}$, which makes the relative origin asymmetric. From Equation 22 , it follows that $\phi=\mathrm{e}^{-\lambda}$ in the limit, when $b_{i k} \rightarrow 1$, and it is therefore natural to talk about an absolute origin $\left(\mathrm{O}_{\mathrm{a}}\right)$ :

$$
\mathrm{O}_{\mathrm{a}}=\left(\phi_{\mathrm{i}}, \phi_{\mathrm{k}}\right)=\left(\mathrm{e}^{-\lambda}, \mathrm{e}^{-\lambda}\right),
$$

which is independent of substances matched and is only expressed in the absolute constant $\lambda$.

It has been shown above (under Problem) that the symmetric and asymmetric model are identical when $b_{i k} \rightarrow 1$ (Equation 21). It follows that the absolute origin is symmetric,

$$
\mathrm{O}_{\mathrm{a}}=(\mathrm{C}, \mathrm{C})_{\mathrm{s}}=\left(\mathrm{e}^{-\lambda}, \mathrm{e}^{-\lambda}\right)_{\mathrm{as}},
$$

where indices " $s$ " and "as" stand for symmetry and asymmetry, respectively. Furthermore, $\lambda$ is the natural unit of measurement in the asymmetric model.

Thus, judgments may be considered relative. They depend upon the relation of stimulation to the level of the relative origin that is determined by the relative exponent, $b_{i k}$, and the absolute constant, $\lambda$. This interpretation is in congruence with Helson's adaptation level theory (Helson, 1959) insofar as the relative origin is considered to reflect some internal norm or process that guides or determines when two stimuli are to be judged equal and not equal. Furthermore, the relative origin may exceed or fall below the absolute origin, which is determined by the absolute constant, $\lambda$. The absolute origin may therefore be considered as an internal preset adaptation level comparable to the $37^{\circ} \mathrm{C}$ of the thermoregulation system in man.

The asymmetric model implies that cross-adaptation and the so-called regression effects may be considered as indirect phenomena that reflect the fact that perceptions are relative and can be handled within the content of a general psychophysical function. It should at last be mentioned that the model has been demonstrated only for exponents of which most have been less than one. It is possible that the model must be revised for exponents greater than one. A more general model would also have to include some stochastic parameter, or parameters, in order to take into account the variability in data. Such stochastic parameters are suggested to be independent of the regression effect. 
The symmetric model and measurement. The symmetric model is a model of measurement. Given the exponent of the equal-sensation function for two odors, $\mathrm{A}$ and $\mathrm{B}$, and the exponent connecting odor $\mathrm{B}$ and $a$ third odor $C$ in an equal-sensation function, the exponent for the function when odors $\mathrm{A}$ and $\mathrm{C}$ are matched can be predicted. This is due to the property of an origin for the measurement, the constant $C$. The symmetric model therefore implies measurement on a log-ratio scale because a logarithmic transformation of the symmetric model results in a ratio scale. Of course, this model also must hold approximately for exponents greater than one in order to make any sense. However, the model might need a revision in order to make more precise predictions although the more exact model is of an asymmetric, intransitive nature. A revision of the model is proposed in the following. In order to show this, let us shift to another area of scaling concerned with properties of matrices obtained from ratio estimation.

A ratio matrix will meet the requirements for internal consistency if the response ratios, $r_{i k}$, are transitive, i.e., $r_{i k}=r_{i j} \cdot r_{j k}$ (cf. Eisler, 1960; Goude, 1962). An example where these requirements are violated is given by Goude $(1962$, p. 52). The problem was solved when it could be shown that the ratios obtained had to be multiplied by a constant $k$ in order to meet the requirements for internal consistency, i.e., $\mathrm{kr}_{\mathrm{ik}}=\mathrm{kr}_{\mathrm{ij}} \cdot \mathrm{kr}_{\mathrm{jk}}$, which also can be written: $r_{i k}=k \cdot r_{i j} r_{j k}$ (cf. Eisler, 1960). Suppose the same thing holds approximately for exponent matrices which have been shown to be intransitive and asymmetric in the asymmetric model, then,

$$
b_{i k}=k \cdot b_{i j} b_{j k}
$$

and

$$
b_{k i}=k \cdot b_{k j} b_{j i} \text {. }
$$

The ratio between these equations will be independent of $k$ :

$$
\frac{b_{i k}}{b_{k i}}=\frac{b_{i j} \cdot b_{j k}}{b_{k j} \cdot b_{j i}}=\frac{b_{i j}}{b_{j i}} \cdot \frac{b_{j k}}{b_{k j}} .
$$

Now consider the exponent suggested by Stevens and Greenbaum (1966) to be the "true" exponent, i.e., the geometric mean of $b_{i k}$ and $1 / b_{k i}$. It follows, then, immediately from Equation 31 that an exponent so defined will be transitive, symmetric, and reflexive.

The most simple explanation now seems to be that the asymmetric model takes care of the so-called regression effects. From the comparison between the two models, it can be predicted that regression effects will increase as the exponent $b_{i k}$ gets smaller.
In searching the literature, no decisive data on this prediction was found.

\section{THE EXPERIMENTS}

Three experiments were conducted: In Experiment 1 , hydrogen sulfide was matched to five odorants produced through different combustion procedures of animal manure (Lindvall \& Svensson, 1974); in Experiment 2, pyridine was matched to hydrogen sulfide and pyridine was matched to dimethyl disulfide (Svensson \& Liridvall, 1974). In Experiment 3 , pyridine was matched to hydrogen sulfide and dimethyl disulfide. All three experiments are described in the following. The last experiment is described in more detail as it is not published separately. For a more detailed description of the two earlier experiments, see respective papers.

\section{Experiment 1}

\section{Methods}

Five types of odorous gases were produced through combustion of animal manure. The different odorants were obtained from three electrical combustion units (A, D, and E), one gas-fed furnace (B), and one oil-fed combustion unit (C). They were matched to hydrogen sulfide for equal pleasantness. The method of magnitude matching was used. In one of two exposure hoods, the subject was exposed to four fixed concentrations of the combustion odors, one at the time, and in the other hood to varying concentrations of hydrogen sulfide adjustable in 13 discrete steps. The subject was asked to operate the adjuster until she found the different odors in the two hoods subjectively equal with regard to perceived unpleasantness.

The results of the equal unpleasantness matchings were averaged (geometric means) into group data. Every point represents the average of 30 observations, except for combustion gas, C. In this case, the number of observations at each point is only 10 due to technical disturbances during the experiment.

The trend of the equal-sensation function can be represented approximately by straight lines in a $\log -\log$ plot, although the standard deviation in each test point is considerable (see Lindvall \& Svensson, 1974). The apparent linearity in log-log plots indicates an equal-sensation function of the basic form (Equation 1).

\section{Experiment 2}

Odor intensity matchings were obtained for three chemical compounds: hydrogen sulfide, pyridine, and dimethyl disulfide. Four experiments were run: (a) pyridine matched to hydrogen sulfide, (b) dimethyl disulfide matched to hydrogen sulfide, (c) hydrogen sulfide matched to dimethyl disulfide, and (d) pyridine matched to dimethyl disulfide. Four subjects participated in the experiment. It was found that the equal-sensation function for intramodal matchings described the data well (Equation 1). In order to obtain a precise control of the stimulation during matching, a matching method of successive approximations was developed (see Svensson \& Szczygiel, 1974).

The results of the four matching experiments can be seen in Svensson and Lindvall (1974). Every point in each plot is the geometric mean of 64 observations (16 observations per subject). the linearity in these log-log plots is obvious.

\section{Experiment 3}

Subjects. Six subjects participated in the experiment. They were 
students aged 20-30 years without any previous experience of psychological experiments.

Stimuli. The odorous substances were hydrogen sulfide $\left(\mathrm{H}_{2} \mathrm{~S}\right.$; Matheson, technical grade), pyridine (Pyr; Mallinckrodt, analytical reagent), and dimethyl disulfide (DMDS; Eastman, 97, 6\% GLC).

The hydrogen sulfide was kept in gas form in tubes. The gas was taken directly from the tubes and led into the dosage system. Pyridine and dimethyl disulfide were kept in liquid form in impinger tubes. The tubes were submerged in a thermostatically controlled water bath $\left(16^{\circ} \mathrm{C}\right)$, and a continuous stream of nitrogen was swept over the surface of the substances. This flow was regulated so as to obtain a continuous stream of nitrogen laden with the odorous gas.

Chemical analysis. Analysis of hydrogen sulfide was carried out once for every $S$ and session by employing the gel technique method described by Broström and Brosset (1969). Samples were taken immediately in front of the dosing capillaries.

Pyridine and dimethyl disulfide were analyzed with a gas chromatograph (Aerograph $204 \mathrm{~B}$ ). The analyses were run using $5 \%$ Carbowax $20 \mathrm{M} /$ chromosorb $\mathrm{W}$ as a column at $100^{\circ} \mathrm{C}$ and flame ionization detector. The gas chromatograph was calibrated with a dilution of pyridine in water and a dilution of dimethyl disulfide in carbontetrachloride, respectively.

Experimental equipment. The laboratory is constructed as a mobile unit for sensory evaluation of odorous air pollutants. The olfactometer involves a well-defined dynamic flow of gas which permits stable exposure concentrations, rapid changes in concentration, and fairly natural respiratory conditions (Lindvall, 1970). The equipment consists essentially of a ventilated and airconditioned test chamber, in which two Teflon-coated exposure hoods have been installed. The odorant gas is mixed with purified air in fixed dilutions and added to the flow of air into the hoods by means of electromagnetically operated steel and Teflon cannulae of varying bore. A special waiting room for the subjects, with ventilation comparable to the actual test procedure, provides optimal adaptation. By using suitable filter equipment (active charcoal filters and particle filters), it is possible to carry out evaluations with a minimum of contaminations in the laboratory air.

Process control. In order to obtain a precise control of the stimulation during the matchings, a new method of successive approximations with automatc equipment was developed (Svensson \& Szczygiel, 1974). The reference stimulus is presented in one of the hoods and the matching stimulus in the other. The subjects have to decide whether (1) the odor in the left hood is the more intense, (2) the odors in the two hoods are equally intense, or indistinguishable, or (3) the odor in the right hood is the more intense.

The responses are fed into a logic unit by an electrical signal system. The information is then processed and a new matching stimulus is automatically initiated, according to an algorithm that bisects intervals up or down in successive trials.

The subject can make a decision in four trials at most. As soon as the subject signals the second response alternative above, no further matching stimulus is presented, the logic unit stops, and the result is visualized on a panel to the experimenter. If perceived equality is not attained at Trial 4, the result will be reported as being more or less than the nearest stimuli chosen. This strategy is optimal, with four binary decisions to find 1 interval out of 16 possible (see further, Svensson \& Szcyzygiel, 1974).

The judgment situation is easily understood, even by naive subjects, and the strategy is the same for all. Distortion effects due to cognitive, motivational, and learning factors are minimized.

Procedure. In order to validate the equal-sensation function, two experimental combinations, were studied. Pyridine was matched to $\mathrm{H}_{2} \mathrm{~S}$, and $\mathrm{H}_{2} \mathrm{~S}$ to pyridine.

The method of magnitude matching was used. In the left hood, the subjects were exposed to 5 concentrations of the reference odorant ordered in a geometrical progression. In the right hood, the subjects could be presented with any of 15 different concentrations of the matching odorant, also ordered in a geometrical series. The five reference stimuli were presented in random order in every session.

Five absolute thresholds were estimated for every subject and substance before the main experiment started. A first matching experiment was run. Every subject made 16 matchings to every stimulus presented.

After this, another 5 absolute thresholds were estimated. At the end, a new matching session took part with the same conditions as the first. In Table 2, geometric means of the total 10 absolute thresholds for hydrogen sulfide for one of the subjects was not obtained because this subject was not able to perform her task for this special substance. Geometric group mean values are also tabulated.

The reference odorants in milligrams/liter were chosen as

$$
10^{\mathrm{t}} \mathrm{r}+0.5 \mathrm{k}
$$

where $t_{1}$ is $-\log$ threshold concentration and $k=1, \ldots 5$ for the 5 reference stimuli. The 15 matching stimuli were chosen in accordance with the expression.

$$
10 t_{m}+0.25 k
$$

where $t_{m}$ is $-\log$ threshold concentration in milligrams/liter and $k=0, \ldots 14$ for the 15 matching stimuli. By this selection, the range of the matching odorant is intended to cover the range of the reference odorant and thus minimize possible category effects.

A green light inside the hood flashed for $8 \mathrm{sec}$. Within this time, the subject had to make a magnitude match by first taking a sniff in one of the hoods and then quickly turning to the other for another sniff. Between trials, the subject had to wait for $18 \mathrm{sec}$. The subjects were thoroughly instructed and supervised in this task.

The sessions were run according to an ABBA design. The order in which the subject was to smell the stimuli in the two hoods was also balanced, first the reference stimulus and then the matching stimulus, or vice versa.

\section{Results}

In Figure 1, the two equal-sensation plots for group data are shown, with one plot for each experimental condition. Every point in the plots is the geometrical mean of 192 observations with 32 observations per subject. The concentrations are expressed as $\log (\mathrm{S} / \mathrm{So})$, where the numerator $(\mathrm{S})$ is the odor intensity in milligrams/liter air and $\mathrm{S}_{\mathrm{o}}$ is the absolute threshold in the same measure. It can be seen that the linearity in the log-log coordinates is evident. Straight lines are therefore fitted to the data by means of the method of least squares. Thus, in linear coordinates, a power function fits to the data well.

Table 2

Experiment 3: Absolute Thresholds Expressed as - $\log (\mathrm{mg} / 1)$

\begin{tabular}{lccccccr}
\hline \multirow{2}{*}{$\begin{array}{c}\text { Sub- } \\
\text { stance }\end{array}$} & \multicolumn{7}{c}{ Subject } \\
\cline { 2 - 7 } & A & B & C & D & E & F & Mean \\
\hline $\mathrm{H}_{2}$ S & 4.96 & 5.59 & 4.66 & 5.22 & & 4.99 & 5.08 \\
Pyridine & 3.68 & 3.79 & 3.81 & 3.98 & 3.88 & 3.74 & 3.81 \\
DMDS* & 3.97 & 3.98 & 4.01 & 3.87 & 4.12 & 3.45 & 3.90 \\
\hline
\end{tabular}

*DMDS = dimethyl disulfide 

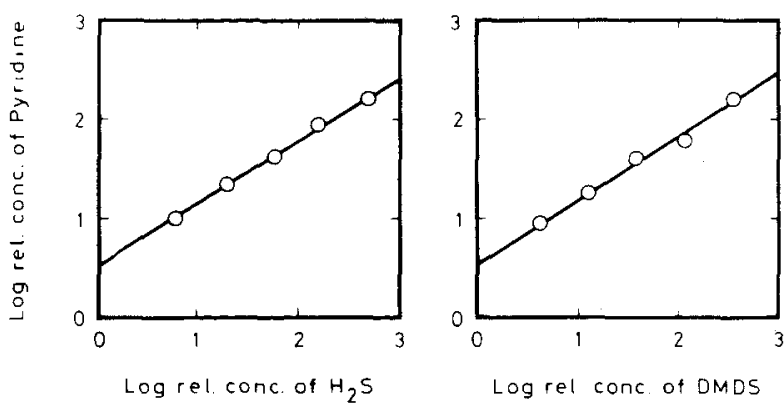

Figure 1. Experiment 3: Equal-sensation functions in $\log \log$ coordinates obtained for group data in two subexperiments: pyridine matched to hydrogen sulfide, and pyridine matched to dimethyl disulfide. The straight lines are fitted by means of the method of least squares. The concentrations are expressed relative to absolute thresholds.

This result is in accordance with what Lindvall and Svensson (1974) and Svensson and Lindvall (1974), Experiments 1 and 2 , found for intramodal unpleasantness matching and intramodal intensity matching, respectively, for the sensory modality of olfaction. The finding is congruent with what earlier has been demonstrated for several modalities. (See, e.g., Cain, 1969; Indow \& Stevens, 1966; Stevens, 1966, 1968).

The power functions for the individual data in Experiments 2 and 3, as well as for the group data in Experiments 1, 2, and 3 were analyzed according to the symmetric and asymmetric models. The ratio $\log a_{i k} /\left(1-b_{i k}\right)$ corresponds to $\log C$ for the symmetric model, which can be seen by taking logarithms in Equation 2. The constant, $\lambda$, in Equation 18 is independent of logarithmic base. The ratio $\log a_{i k} / \log \left(1 / b_{i k}\right)$ thus corresponds to $-\lambda$ for the asymmetric model (Equation 3 ).

In Table $3, \log a_{i k}, 1-b_{i k}, \log \left(1 / b_{i k}\right)$, as well as the constants $\log C$ and $\lambda$ are shown for the group data in the three main experiments. The constants, $\log C$ and $\lambda$, were not calculated for the subexperiments in Experiment 2, where the values for $\log a_{i k}$, $1-b_{i k}$, and $\log \left(1 / b_{i k}\right)$ are very close to zero. The calculated values for the constants, $\log C$ and $\lambda$, deviates from the general trend in only one case, namely in Experiment 1 for combustion procedure $\mathrm{E}$. The finding that the ratios $\log a_{i k} /\left(1-b_{i k}\right)$ and $\log a_{i k} / \log \left(1 / b_{i k}\right)$ are approximately constant indicates that Equations 2 and 3 both satisfactorily describe the functional relationship between the multiplicative constant and the exponent of the equal-sensation function.

In order to make a more accurate analysis of data, $\log a_{i k}$ was plotted against $b_{i k}$ and $\log \left(1 / b_{i k}\right)$ for the group data (all three experiments) as well as for the individual data in Experiments 2 and 3. These plots are shown in Figures 2 and 3. Diagram $A$ in these figures shows the results for the individual data (open circles) as well as for the group data (filled circles). In order to show better the trend for the group data, it was also plotted in the double scale (Figures 2 and 3, Diagram B).

Regression analyses were run for both the symmetric and asymmetric models. The outcome of the analysis can be judged from Tables 4 and 5. The intercepts, $\alpha$, are close to zero for both models. Furthermore, the constant $-\lambda$ in the asymmetric model and the constant $\log \mathrm{C}$ in the symmetric model are both significant at the .0001 level with standard error of the means of the same order of magnitude. The correlations are also of the same order of magnitude and of course also the proportion of variance accounted for by regression. However, the variance explained is slightly higher for the asymmetric model. The linearity for the two models was also tested with polynomial regression. No significant higher order terms of $\log 1 / b_{i k}$ for the asymmetrical model for either individual data or group data came out from the analysis, this fact lending support to the model. For the symmetric model, a second-order polynomial in $\left(1-b_{i k}\right)$ was significant for group data $p<.0001$ but not for individual data, this fact indicating that this model may be inferior to the asymmetric model. However, deviations from linearity could not be demonstrated for both individual and group data. To summarize, it is hard to judge from the present data which model is the better from a statistical point of view, even though the possibility that the asymmetric model is the better has been shown.

Table 3

Estimates of Constants in the Symmetric and Asymmetric Models

\begin{tabular}{|c|c|c|c|c|c|c|}
\hline \multicolumn{2}{|c|}{ Substance } & \multirow{2}{*}{$\begin{array}{l}\log \\
\mathbf{a}_{\mathbf{i k}}\end{array}$} & \multirow[b]{2}{*}{$1-\mathbf{b}_{\mathbf{i k}}$} & \multirow{2}{*}{$\begin{array}{c}\log \\
1 / \mathbf{b}_{\mathbf{i k}} \\
\end{array}$} & \multirow{2}{*}{$\begin{array}{l}\log \\
\mathrm{C}^{*}\end{array}$} & \multirow[b]{2}{*}{$\lambda^{* *}$} \\
\hline Matching & Reference & & & & & \\
\hline \multicolumn{7}{|c|}{ Experiment 1} \\
\hline $\begin{array}{l}\mathrm{H}_{2} \mathrm{~S} \\
\mathrm{H}_{2} \mathrm{~S} \\
\mathrm{H}_{2} \mathrm{~S} \\
\mathrm{H}_{2} \mathrm{~S} \\
\mathrm{H}_{2} \mathrm{~S}\end{array}$ & $\begin{array}{l}\mathrm{A}^{\dagger} \\
\mathrm{B} \dagger \\
\mathrm{C}^{\dagger} \\
\mathrm{D} \dagger \\
\mathrm{E} \dagger\end{array}$ & $\begin{array}{l}.729 \\
.879 \\
.952 \\
.963 \\
.532\end{array}$ & $\begin{array}{l}.460 \\
.530 \\
.560 \\
.567 \\
.608\end{array}$ & $\begin{array}{l}.268 \\
.328 \\
.357 \\
.363 \\
.408\end{array}$ & $\begin{array}{r}1.58 \\
1.66 \\
1.70 \\
1.70 \\
.88\end{array}$ & $\begin{array}{l}2.72 \\
2.68 \\
2.67 \\
2.65 \\
1.30\end{array}$ \\
\hline \multicolumn{7}{|c|}{ Experiment 2} \\
\hline $\begin{array}{l}\mathrm{H}_{2} \mathrm{~S} \\
\text { DMDS } \dagger \dagger \\
\text { Pyridine } \\
\text { Pyridine } \\
\text { Pyridine } \\
\text { Pyridine }\end{array}$ & $\begin{array}{l}\text { DMDS } \\
\mathrm{H}_{2} \mathrm{~S} \\
\text { DMDS } \\
\mathrm{H}_{2} \mathrm{~S} \\
\text { DMDS } \\
\mathrm{H}_{2} \mathrm{~S}\end{array}$ & $\begin{array}{l}.034 \\
.040 \\
.467 \\
.596 \\
.539 \\
.529\end{array}$ & $\begin{array}{r}-.060 \\
-.026 \\
.325 \\
.400 \\
.366 \\
.393\end{array}$ & $\begin{array}{r}-.025 \\
-.011 \\
.171 \\
.222 \\
.198 \\
.217\end{array}$ & $\begin{array}{l}1.44 \\
1.49 \\
1.47 \\
1.64\end{array}$ & $\begin{array}{l}2.73 \\
2.68 \\
2.72 \\
2.44\end{array}$ \\
\hline
\end{tabular}

${ }^{*} \log C=\left(\log a_{i k}\right) \dot{(}\left(1-b_{i k}\right)$, the symmetric model (cf. Equation 2).

${ }^{*} \log C=\left(\log a_{i k}\right) /\left(\log 1 / b_{i k}\right)$, the asymmetric model $/ c f$. Equation 3).

fOdorant produced by combustion in: $A$, an electrical combustion unit; $B$, a gas-fed furnace; $C$, an oil-fed combustion unit; $D$, an electrical combustion unit; $E$, an electrical combustion unit.

††DMDS $=$ dimethyl disulfide 

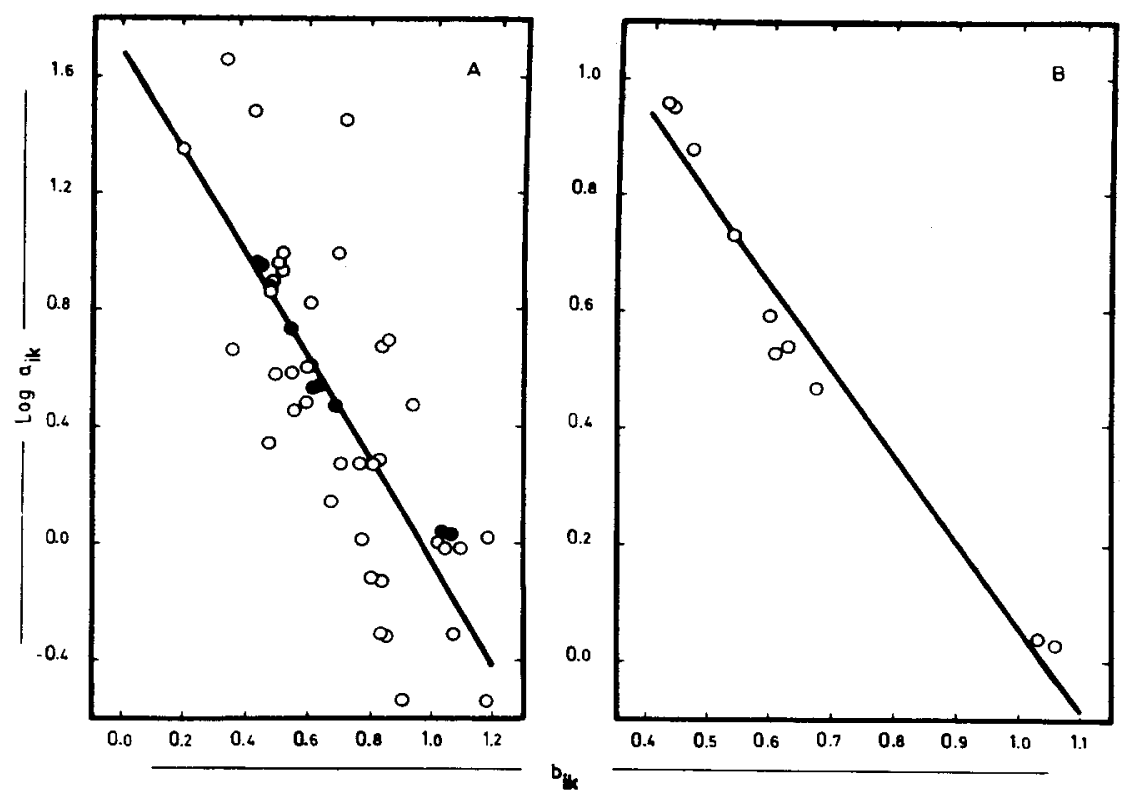

Figure 2. The symmetric model. In diagram $A, \log a_{i k}$ is plotted against $b_{i k}$ for the individual data in Experiments 2 and 3 (open circles) and group data from Experiments 1, 2, and 3 (filled circles). The regression line fitted to $\log a_{i k}$ as a function of $1-b_{i k}$ for the data from the individuals is: intercept, $\alpha=-0.07$; slope, $\log C=1.75$. In diagram $B, \log a_{i k}$ is plotted against $b_{i k}$ in the double scale for the group data. The regression line for $\log a_{i k}$ as a function of $1-b_{i k}$ is: intercept, $\alpha=0.06$; slope, $\log \mathrm{C}=1.46$.

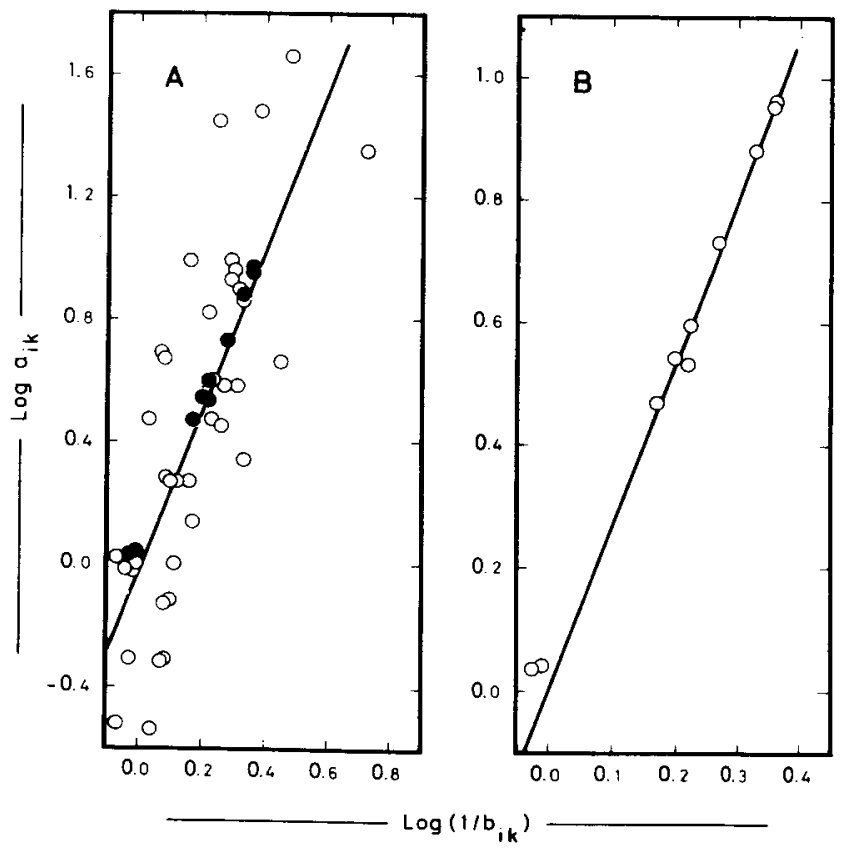

Figure 3. The asymmetric model. In diagram $A, \log a_{i k}$ is plotted against $\log \left(1 / b_{i k}\right)$ for the individual data in Experiments 2 and 3 (open circles) and group data from Experiments 1, 2, and 3 (filled circles). The regression line is fitted to the data points for the individuals by means of the method of least squares. (Intercept, $\alpha=-0.02$; slope, $-\lambda=2.57$.) In diagram $B, \log a_{i k}$ is plotted against $\log \left(1 / b_{i k}\right)$ in the double scale for the group data. (Intercept, $\alpha=0.04$; slope, $-\lambda=2.50$.)

\section{DISCUSSION}

\section{Cross-Adaptation}

Engen (1963) tried to find out whether chemicals similar in some physicochemical properties also show more cross-adaptation than those chemicals that are less similar. For this matter, he chose a homologous series of alcohols where many of the physical properties vary in an orderly fashion with carbon chains in these molecules. Engen's hypothesis was that the closer the members in homologous series are, the more they will cross-adapt each other. The experiment, however, gave no support to his prediction. But the critical point may be how similar the odorants are from a psychological point of view, not a physical one. In the equal-sensation function, the exponent reflects some kind of "similarity." The more "similar" the odorants are, the more close to one will the exponent be. It is suggested that the "similarity" as reflected by the exponent covaries with qualitative similarity. But the prediction, then, from Equation 24 is in the opposite direction from that suggested by Engen, i.e., the more similar the odorants are psychologically, the less they will crossadapt (see Equations 22, and 24). Cross-adaptation also manifests itself in an asymmetrical effect, i.e., given two odorants, $\mathrm{A}$ and $\mathrm{B}$, odorant $\mathrm{A}$ will crossadapt odorant B more than odorant B will crossadapt odorant $A$, or vice versa. This effect has been noted by several investigators for different 
Table 4

Fit of the Symmetric Model

\begin{tabular}{lcrccc}
\hline Data Set & $\mathrm{N}$ & $\alpha$ & $\log \mathrm{C}^{*}$ & $\mathrm{r}$ & $\mathrm{r}^{2}+$ \\
\hline Individual & 38 & -.07 & $1.75 \pm .04$ & .753 & .567 \\
Group & 10 & .06 & $1.46 \pm .03$ & .981 & .962 \\
\hline
\end{tabular}

* Values of $\log C$ are given \pm the standard error of the mean: $p<.0001$.

tProportion of variance accounted for by regression.

Table 5

Fit of the Asymmetric Model

\begin{tabular}{lccccc}
\hline Data Set & $\mathrm{N}$ & $\alpha$ & $-\lambda^{*}$ & $\mathrm{r}$ & $\mathrm{r}^{2}$ \\
\hline Individual & 38 & -.02 & $2.57 \pm .06$ & .776 & .595 \\
Group & 10 & .04 & $2.50 \pm .03$ & .996 & .992 \\
\hline
\end{tabular}

*Values of $-\lambda$ are given \pm the standard error of the mean; $p<.0001$.

tProportion of variance accounted for by regression.

Table 6

Ranges for Reference Stimuli

\begin{tabular}{lccc}
$\begin{array}{l}\text { Reference } \\
\text { Substance }\end{array}$ & $\log \mathrm{S}_{\min } / \mathrm{S}_{\circ}$ & $\log \mathrm{S}_{\max } / \mathrm{S}_{\circ} \log \mathrm{S}_{\max } / \mathrm{S}_{\min }$ \\
\hline & \multicolumn{3}{c}{ Experiment 1} \\
$\mathrm{~A}$ & .19 & 1.69 & 1.50 \\
$\mathrm{~B}$ & .24 & 1.82 & 1.58 \\
$\mathrm{C}$ & .18 & 1.73 & 1.50 \\
$\mathrm{D}$ & .19 & 1.76 & 1.58 \\
$\mathrm{E}$ & .19 & 1.73 & 1.54 \\
& & Experiment 2 & \\
DMDS* & .47 & 2.44 & 1.97 \\
$\mathrm{H}_{2} \mathrm{~S}$ & .57 & 2.54 & 1.97 \\
DMDS & .49 & 2.46 & 1.97 \\
$\mathrm{H}_{2} \mathrm{~S}$ & .66 & 2.63 & 1.97 \\
& & Experiment 3 & \\
DMDS & .81 & 2.71 & 1.90 \\
$\mathrm{H}_{2} \mathrm{~S}$ & .63 & 2.55 & 1.92 \\
\hline
\end{tabular}

${ }^{*} D M D S=$ dimethyl disulfide.

pairs of odorants (cf. Cain, 1970; Corbit, 1969; Engen, 1963; Engen \& Bosack, 1969; Moncrieff, 1956).

The symmetric model, on the other hand, must be considered insensitive of explaining crossadaptation. For this case, the experimental threshold (see Equation 25) is directly proportional to the absolute threshold, where the proportionality constant is invariant of substances matched. Furthermore, a symmetric model (e.g., Equation 23) never will be able to explain the asymmetric effect demonstrated for cross-adaptation.

\section{Odor Quality}

The calculated constants $-\lambda$ and $\log C$ for the two models for group data in Experiment 1, combustion procedure $\mathrm{E}$, deviated from the general trend. In this case, the constants were about half of that ob- tained in the other experiments (see Table 3 ). The separation of this point from the general trend may depend on disturbances in the olfactometer. Another explanation is of perceptual origin. It is thus possible that odors cluster into groups and that the obtained constants, $\lambda$ and $\log C$, vary between groups. The quality of the odors within these groups may be of similar character. This shoud mean that the constants vary with quality. A model of this kind has been suggested by Berglund, Berglund, Lindvall, and Svensson (1973) for the perception of odor mixtures. However, the exponents in the equal-sensation function will be close to one for odorants that are "similar." It is thus possible that the equal-sensation function takes care of qualitative aspects as reflected by the exponent (cf. former section).

\section{Range Effects}

The interpretations according to the asymmetric model must be considered independent of range effects discussed by Teghtsoonian (1973). From Table 6, it can be seen that the range of reference stimuli in Experiment 1 is rather constant, with a value of ca. 1.5 and ca. 2.0 in Experiments 2 and 3, where the ranges are measured as $\log \left(\mathrm{S}_{\max } / \mathrm{S}_{\min }\right)$. Also $\log \left(\mathrm{S}_{\max } / \mathrm{S}_{\mathrm{o}}\right)$ and $\log \left(\mathrm{S}_{\min } / \mathrm{S}_{\mathrm{o}}\right)$, where $\mathrm{S}_{\mathrm{o}}$ is absolute threshold are tabled.

For the group data from the three main experiments with different ranges (1.5 in Experiment 1 and 2.0 in Experiments 2 and 3), the fit of the asymmetric model was more than satisfactory, with $99.2 \%$ of the variance explained by linear regression. Furthermore, no significant higher order polynomial could be demonstrated.

It is important to note that the results were obtained despite the fact that the range in Experiment 1 is a little smaller than that in Experiments 2 and 3. Of course, a possible alternative explanation is that these ranges are too close in order to display any differences in range effects. The symmetric model, however, may be dependent on range effects. This conclusion is based on the fact that significant deviation from linearity could be demonstrated for the model in linearized coordinates. But it must also be remembered that $96.2 \%$ of the total variance was explained by the model, despite the fact that data from experiments with different ranges were included in the analysis.

\section{REFERENCE NOTE}

1. Reese. T. S., Kryter, K. D., \& Stevens, S. S. The relative annoyance produced by various bands of noise. Psychoacoustic Laboratory, Harvard University, IC-65, 17 March 1944.

\section{REFERENCES}

Berglund, $\ddot{B}$. An analysis of some basic mechanisms of sensory perception with direct scaling methods. Reports from the Psycho- 
logical Laboratories. The University of Stockholm, 1971. Suppl. 6.

Berglund. B.. Berglund. U.. Lindvall, T., \& Svensson, L. T. A quantitative principle of perceived intensity summation in odor mixtures. Journal of Experimental Psychology. 1973. 100. 29-38.

Broström. C. E.. \& Brosset. C. A. A method for simultaneous determination of hydrogen sulfide and sulfur dioxide in flue gases. Atmospheric Environment, 1969, 3, 407-416.

CaIN. W. S. Odor intensity: Differences in the exponent of the psychophysical function. Perception \& Psychophysics, 1969. 6. 349-354.

CaIN. W. S. Odor intensity after self-adaptation and crossadaptation. Perception \& Psychophysics, 1970, 7, 271-275.

Cor вIт. T. E. Facilitation of olfactory signal detection by crossadaptation. Unpublished doctoral dissertation. Brown University. 1969.

EISLER. H. Similarity in the continuum of heaviness with some methodological and theoretical considerations. Scandinavian Journal of Psychology, 1960, 1. 69-81.

Engen. T. Cross-adaptation to the aliphatic alcohols. American Journal of Psychology, 1963, 76, 96-102.

ENGEN. T., \& Bosack, T. N. Facilitation in olfactory detection. Journal of Comparative and Physiological Psychology. 1969. 68. $320-326$.

GOUDE. G. On fundamental measurement in psychology. Stockholm: Almquist \& Wiksell. 1962.

Helson. H. Adaptation level theory. In S. Koch (Ed.), Psychology, a study of a science (Vol. 1). Sensory, perceptual and psychological foundations. New York: McGraw-Hill, 1959. Pp. 565-621.

Hempel. C. G. Philosophy of natural science. London: PrenticeHall. 1966.

In Dow. T., \& Stevens, S. S. Scaling of saturation and hue. Perception \& Psychophysics, 1966, 1, 253-271.

Krantz. D. H., LuCe, R. D., Suppes, P., \& Tversky, A. Foundations of measurement. New York: Academic Press. 1971.
Kryter. K. D.. \& Pearsons. K. S. Some effects of spectral content and duration on perceived noise level. Journal of the Acoustical Society of America, 1963, 35, 866-883.

Lindvale, T. On sensory evaluation of odorous air pollutant intensities. Nordisk Hygienish Tidskrift, 1970. Suppl. 2, 1-181.

Lindvall, T., \& Svensson, L. T. Equal-unpleasantness matching of malodorous substances in the community. Journal of Applied Psychology, 1974, 59, 264-269.

MONCRIEFF, R. W. Olfactory adaptation and odour likeness. Journal of Physiology, 1956, 133, 301-316.

Stevens, S. S. On the new psychophysics. Scandinavian Journal of Psychology, 1960, 1, 27-35.

Stevens, S. S. Power-group transformations under glare, masking. and recruitment. Journal of the Acoustical Society of America, 1966, 39, 725-735.

Stevens, S. S. Tactile vibration: Changes of exponant with frequency. Perception \& Psychophysics, 1968, 3, 223-228.

STEVENS. S. S. On the quantitative evaluation of noise. In J. D. Chalupnik (Ed.). Transportation noises. Seattle and London: University of Washington Press, 1970. Pp. 114-128.

Stevens, S. S., \& Greenbaum. H. B. Regression effects in psychophysical judgment. Perception \& Psychophysics. 1966, 1. 439-446.

Svensson, L. T.. \& Lindvall, T. On the validity of intramodal intensity matching in olfaction. Perception \& Psychophysics, 1974, 16, 264-270.

Svensson. L. T., \& Szczygiel, K. A matching method of successive approximations and its instrumentation. Behavioral $R e$ search Methods \& Instrumentation, 1974, 6, 13-18.

TEghtsoonian, R. Range effects in psychophysical scaling and a revision of Steven's law. American Journal of Psychology, 1973, 86. $3-27$.

(Received for publication January 17, 1977; revision accepted March 31, 1977.) 